\title{
Una nota sobre valoración de opciones financieras y ecuaciones diferenciales parciales no lineales (I)
}

\section{A note about option pricing and nonlinear partial differential equations (I)}

John Freddy Moreno Trujillo*

* Estudiante de Doctorado en Ciencias Económicas. Magíster en Matemática Aplicada. Docente-Investigador, CIPE-ODEON, Universidad Externado de Colombia, Bogotá (Colombia). [jhon.moreno@uexternado.edu.co].

Artículo recibido el 15 de diciembre de 2018.

Aceptado el 26 de diciembre de 2018.

Para citar este artículo

Moreno Trujillo, J. F. (2018). Una nota sobre valoración de opciones financieras y ecuaciones diferenciales parciales no lineales (I). ODEON, 15, 53-71.

DOI: https://doi.org/10.18601/17941113.n15.03 


\section{Introducción}

Modelos clásicos de valoración de opciones financieras como el binomial de Cox, Ross y Rubinstein (1979) o el de Black y Scholes (1973) están basados en una serie de supuestos simplificadores que permiten establecer expresiones analíticas o semianalíticas para el valor de la prima de la opción considerada. De igual forma, los procedimientos de aproximación numérica como Monte Carlo o diferencias finitas se aplican a partir de una serie de supuestos sobre las variables financieras relacionadas con el ejercicio de valoración.

Algunos de los supuestos más comunes son: volatilidades constantes asociadas al comportamiento del precio del activo subyacente, ausencia de costos de transacción, mercados perfectamente líquidos, tasas de interés iguales para inversión o préstamo y ausencia de riesgo de contraparte.

Al revisar la literatura refrente a la valoración de derivados financieros se pueden encontrar múltiples trabajos en los cuales se desarrollan procedimientos o expresiones de valoración que eliminan uno o varios de estos supuestos, por ejemplo, Avellaneda, Levy y Parás (1995), Lyons (1995) y Carmona y Nadtochiy (2009) introducen modelos de valoración con volatilidades inciertas; en Leland (1985) se desarrolla un modelo con costos de transacción, que es extendido en trabajos como los de Kampen y Avellaneda (2003) o Forsyth (2001). En Benhamou, Rivoira y Gruz (2008) se consideran tasas de interés no determinísticas en modelos de volatilidad local incierta, y en Cetin, Jarrow y Protter (2004) y Cetin, Soner y Touzi (2010) se estudia el problema de valoración en mercados ilíquidos con restricciones Gamma.

Referencias como las anteriores son solo una muestra del interés que despierta, tanto desde la perspectiva práctica como desde la académica, el problema de desarrollar modelos de valoración de derivados (en particular de opciones), sin acudir a supuestos alejados del comportamiento real de los mercados.

Un elemento muy frecuente, si es que no común, en los modelos de valoración menos restrictivos en sus supuestos, es que como parte del proceso de valoración se llega al problema de resolver una ecuación diferencial parcial (EDP) semilineal o totalmente no lineal. Esto como resultado de extender los procedimientos de valoración clásicos que están basados en un enfoque de EDP, al caso en el cual alguno o algunos de los supuestos es eliminado.

El presente documento es el primero de una serie de trabajos en los cuales se desarrollarán en detalle los elementos necesarios para llegar a la valoración no lineal de instrumentos financieros derivados, desde la perspectiva de EDP. Es 
importante aclarar que la resolución de EDP semilineales o no lineales puede no ser posible en forma analítica, e incluso las aproximaciones numéricas pueden resultar ineficientes, lo que hace al tema de interés dada la posibilidad abierta de nuevos procedimientos de solución.

En este primer trabajo se da una visión general de los problemas de valoración no lineal que serán considerados. Para esto, en la primera sección se presenta el modelo de mercado financiero y el problema genérico de valoración bajo los supuestos clásicos, junto con los detalles del modelo Black-Scholes de valoración como ejemplo. Se recomienda a los lectores que no están familiarizados con los resultados básicos de cálculo estocástico referirse a Moreno Trujillo (2015) y Venegas Martínez (2008). En la sección 2 se hace una presentación breve del contexto general de las EDP de segundo orden no lineales, y la relación de este tipo de ecuaciones con los problemas de control óptimo estocástico y la EDP de Hamilton-Jacobi-Bellman.

En la sección 3 se presentan los detalles iniciales de los problemas no lineales de valoración en finanzas que serán estudiados a lo largo de esta serie de trabajos. Por último se presentan las conclusiones y extensiones.

\section{Modelo de mercado}

Se considera un espacio de probabilidad filtrado $\left(\Omega,\left(F_{t}\right)_{0 \leq t \leq T}, P\right)$, donde $P$ es una medida de probabilidad histórica (probabilidad de mercado), sobre el cual se definen los procesos estocásticos $S_{t}^{i}$, con $i=1,2, \ldots, n$ que describen el precio de los activos riesgosos de la economía. Estos procesos satisfacen la ecuación diferencial estocástica multidimensional:

$$
d S_{t}^{i}=\mu_{i}\left(t, S_{t}\right) d t+\sum_{j=1}^{d} \sigma_{i, j}\left(t, S_{t}\right) d W_{t}^{j}
$$

donde: $\mu_{i}\left(t, S_{t}\right)$ denota la tasa de rentabilidad instantánea del activo, $\sigma_{i, j}\left(t, S_{t}\right)$ es el elemento en la fila $i$-columna $j$ de la matriz de varianzas y covarianzas de los activos considerados, y $W_{t}^{j}$ es un movimiento Browniano estándar que describe la fuente de incertidumbre del activo $j$. Adicionalmente, se considera un activo de valor en $t$ denotado por $B_{t}$, que representa el valor dinero en el mercado, y que satisface la ecuación: 


$$
d B_{t}=r_{t} B_{t} d_{t}, B_{0}=1
$$

donde: $r_{t}$ es la tasa corta de interés, que puede asumirse determinística o estocástica ${ }^{1}$. Los portafolios de activos tienen valor en $t$ denotado por $\Pi_{t}$, y son autofinanciados si satisfacen:

$$
d \bar{\Pi}_{t}=\sum_{i=1}^{m} \Delta_{t}^{i} d \bar{S}_{t}^{i}
$$

donde: $\Delta_{t}^{i}$ denota el número de unidades que se tiene del activo $i$ el instante $t$. Se considera en este mercado un derivado con vencimiento en $T$ y posible valor intrínseco $F_{T}$, que depende del precio del activo o activos subyacentes $S_{t}^{1}, S_{t}^{2}, \ldots$, $S_{t}^{m} \operatorname{con} t \leq T$.

Como es conocido, si el mercado es completo existe una medida de probabilidad $Q$ equivalente $P^{2}$ tal que el valor en $t \leq T$ del derivado está dado por:

$$
E^{Q}\left[D_{t T} F_{T} \mid F_{t}\right]
$$

donde: $E^{Q}\left[\cdot \mid F_{t}\right]$ denota el valor esperado condicional bajo la medida $Q$. Lo anterior se tiene porque si el mercado es completo, el valor del portafolio de superreplicación del comprador descontado, asumiendo que el valor pagado por el derivado al momento de su negociación inicial es $z$, está dado por:

$$
\sup \left\{z \in F_{t} \mid \bar{\Pi}_{T}^{C}=-D_{0 t} z+\sum_{j=1}^{m} \int_{t}^{T} \Delta_{s}^{i} d \bar{S}_{u}^{i}+D_{0 T} F_{T} \geq 0\right\}
$$

esto considerando que una cobertura delta constituida por el derivado y la posición en los activos subyacentes al mismo es igual al valor del portafolio descontado de superreplicación del vendedor 1 A partir de este activo se define el proceso de descuento entre 0 y $t$ como $D_{0 t}=\exp \left\{-\int_{0}^{t} r_{s} d s\right\}$,

2 Las medidas de probabilidad $P$ y $Q$ se dicen equivalentes si $Q(A)=0$ si y solo $P(A)=0$. 


$$
\inf \left\{z \in F_{t} \mid \bar{\Pi}_{T}^{V}=D_{0 t} z+\sum_{j=1}^{m} \int_{t}^{T} \Delta_{s}^{i} d \bar{S}_{u}^{i}-D_{0 T} F_{T} \geq 0\right\}
$$

Si en general $r_{t}=\left(t, S_{t}\right)$ y $F_{T}=g\left(S_{T}\right)$ entonces:

$$
u\left(t, S_{t}\right)=E^{Q}\left[\exp \left(-\int_{t}^{T} r\left(u, S_{u}\right) d u\right) g\left(S_{T}\right) \mid F_{t}\right]
$$

es la función que describe el valor del derivado en el instante $t$, dado que el valor del activo riesgoso subyacente en este instante es $S_{t}$. La función $u(t, x)$ asociada a la ecuacion (7) satisface la ecuación diferencial parcial:

$$
\partial_{t} u(t, x)+A u(t, x)-r(t, x) u(t, x)=0 \quad ; \quad u(T, x)=g(x)
$$

donde: $A(\cdot)$ es el generador de Itô ${ }^{3}$ del proceso $S^{i}$. Para EDP de segundo orden lineales, como la expresada en (8), se puede obtener una aproximación estocástica a la solución de la ecuación aplicando el teorema de Feynman-Kac ${ }^{4}$, de forma que:

$$
u(t, x)=E^{Q}\left[\int_{t}^{T} \exp \left(-\int_{t}^{T} r\left(u, S_{u}\right) d u\right) g\left(S_{T}\right) \mid S_{t}=x\right]
$$

3 El generador de Itô se define como:

$$
A=\sum_{i=1}^{n} \mu_{i}(t, x) \partial_{i}+\frac{1}{2} \sum_{i=1, j=1}^{n} \sum_{k=1}^{d} \sigma_{i, k}(t, x) \sigma_{j, k}(t, x) \partial_{i, j}
$$

4 Asumiendo que $\mathrm{F}$ es una solución del problema de Cauchy:

$$
\left\{\begin{array}{l}
\frac{\partial F}{\partial t}+\mu(t, x) \frac{\partial F}{\partial x}+\frac{1}{2} \sigma^{2}(t, x) \frac{\partial^{2} F}{\partial x^{2}}-r F(t, x)=0 \\
F(T, x)=\Phi(x)
\end{array}\right.
$$

y se asume además que $e^{-r s} \sigma(s, X s) \frac{\partial F}{\partial X_{s}}$ es cuadrado integrable, donde X satisface la EDE:

$d X_{s}=\mu\left(s, X_{s}\right) d s+\sigma\left(s, X_{s}\right) d W_{s} \quad ; \quad X_{t}=x$

entonces $F$ tiene representación estocástica:

$$
F(t, x)=e^{-r(T-t)} E_{t, x}\left[\Phi\left(X_{T}\right)\right]
$$




\subsection{El modelo Black-Scholes}

Como un ejemplo de lo anterior se considera el modelo propuesto en 1973 por Fischer Black y Myron Scholes para la valoración de opciones europeas sobre una acción que no paga dividendos y cuyo precio es descrito por un movimiento Browniano geométrico. A continuación se describe cómo llegar a la denominada ecuación diferencial parcial de Black-Scholes. Esta es una EDP de segundo orden (parabólica) y su solución determina el precio de una opción europea si la condición de frontera es el valor intrínseco de la opción. Es importante enfatizar que la solución de la EDP para diferentes condiciones de frontera permite establecer los precios de distintos derivados financieros disponibles en el mercado.

Los supuestos básicos del modelo son:

- El activo subyacente no paga dividendos durante la vida de la opción.

- El precio del activo subyacente $\left(S_{t}\right)$ es descrito mediante un movimiento geométrico Browniano, es decir, satiface la ecuación diferencial estocástica:

$$
d S_{t}=\mu S_{t}+\sigma S_{t} d W_{t}
$$

- La volatilidad del precio del activo es constante en el tiempo ( $\sigma$ constante).

- Existe un mercado de crédito en el que los agentes pueden invertir o prestar a una tasa de interés constante a todos los plazos y libre de riesgo de incumplimiento $(r)$ compuesta continuamente. De acuerdo con esto, si un agente invierte o toma prestado $B_{0}$ unidades monetarias, el cambio en el valor de esta inversión o préstamo en un intervalo de longitud $d t$ satisface:

$$
d B_{t}=r B_{t} d t
$$

- Las ventas en corto del subyacente son permitidas.

- El mercado del subyacente es líquido y divisible, es decir, el subyacente se puede comprar y vender en cualquier fracción de unidad.

- No hay costos de transacción o tasas impositivas.

- En el mercado no existen oportunidades de arbitraje. 
Se considera una opción europea pactada sobre el activo riesgoso con valor en $t$ denotado por $u\left(t, S_{t}\right)$. La aplicación directa del lema de Itô $\hat{o}^{5}$ permite establecer una expresión para el cambio marginal en el valor de la opción sobre el intervalo $[t, t+d t]$.

$$
d u\left(t, S_{t}\right)=\left(\frac{\partial u}{\partial u} \mu S_{t}+\frac{\partial u}{\partial S_{t}}+\frac{1}{2} \sigma^{2} S_{t}^{2} \frac{\partial^{2} u}{\partial S_{t}^{2}}\right) d t+\sigma S_{t} \frac{\partial u}{\partial S_{t}} d W_{t}
$$

Se conforma un portafolio con $\Delta_{1}$ unidades del activo subyacente $\left(S_{t}\right)$ y $\Delta_{2}$ unidades de la opción $\left(u\left(t, S_{t}\right)\right)$, de forma que su valor en $t$ es:

$$
\Pi_{t}=\Delta_{1} S_{t}+\Delta_{2} u\left(t, S_{t}\right)
$$

Por la condición de autofinanciamiento

$$
d \Pi_{t}=\Delta_{1} d S_{t}+\Delta_{2} d u\left(t, S_{t}\right)
$$

y por el supuesto de movimiento Browniano geométrico del precio del activo y la ecuación (10) se tiene que:

$$
d \Pi_{t}=\left(\Delta_{1}+\Delta_{2} \frac{\partial u}{\partial S_{t}}\right) \mu S_{t} d t+\left(\Delta_{1}+\Delta_{2} \frac{\partial u}{\partial S_{t}}\right) \sigma S_{t} d W_{t}+\Delta_{2}\left(\frac{\partial u}{\partial t}+\frac{1}{2} \frac{\partial^{2} u}{\partial S_{t}^{2}} \sigma^{2} S_{t}^{2}\right) d t
$$

El riesgo de mercado, representado por el término asociado a $d W_{t}$, se puede eliminar si se hace una selección adecuada de $\Delta_{1}$ y $\Delta_{2}$. En particular, si $\Delta_{2}=1 \mathrm{y}$ $\Delta_{1}=-\frac{\partial u}{\partial S_{t}}$ (cobertura delta), se tiene que

$$
d \Pi_{t}=\left(\frac{\partial u}{\partial t}+\frac{1}{2} \frac{\partial^{2} u}{\partial S_{t}^{2}} \sigma^{2} S_{t}^{2}\right) d t
$$

5 Dada una función $f(t, x) \in C^{1,2}(\mathbb{R})$ y un proceso estocástico de Itô $X_{t}$, se tiene que

$$
d f\left(t, X_{t}\right)=\frac{\partial f}{\partial t} d t+\frac{\partial f}{\partial X_{t}} d X_{t}+\frac{1}{2} \frac{\partial^{2} f}{\partial X_{t}^{2}}\left(d X_{t}\right)^{2}
$$


Ahora, si el valor inicial del portafolio, conformado siguiendo el esquema de cobertura delta, es decir, $\Pi_{t}=\Delta_{1} S_{t}+\Delta_{2} u\left(t, S_{t}\right)=-\frac{\partial u}{\partial S_{t}} S_{t}+u\left(t, S_{t}\right)$, se invierte a la tasa $r$, se tiene que:

$$
d \Pi_{t}=r\left(-\frac{\partial u}{\partial S_{t}} S_{t}+u\left(t, S_{t}\right)\right) d t
$$

Si en el mercado no existen oportunidades de arbitraje, que es uno de los supuestos del modelo, se debe tener que (14) y (15) son iguales, de donde:

$$
\frac{\partial u}{\partial t}+\frac{1}{2} \frac{\partial^{2} u}{\partial S_{t}^{2}} \sigma^{2} S_{t}^{2}+r S_{T} \frac{\partial u}{\partial S_{t}}-r u\left(t, S_{t}\right)=0
$$

que es la EDP de Black-Scholes, en donde las condiciones de frontera para determinar una solución única están dadas por $u(t, 0)=0$ y $u\left(T, S_{T}\right)=F_{T}$, con $F_{T}$ el valor intrínseco de la opción call o put europea. La ecuación (16) es una ecuación diferencial parcial lineal parabólica, en donde la linealidad significa que si se tienen dos soluciones, entonces la suma de ellas también es una solución. En otras palabras, si todos los activos de un portafolio satisfacen esta ecuación, entonces el portafolio también la satisface.

La aplicación del teorema de Feynman-Kac a la ecuación (16) lleva la expresión:

$$
u(t, x)=e^{-r(T-t)} E^{Q}\left[F_{T}=g\left(S_{T}\right) \mid S_{t}=x\right]
$$

que es un caso particular del primer teorema fundamental de valoración de activos contingentes.

\section{EDP no lineales}

En términos generales, una ecuación diferencial parcial completamente no lineal, asociada a una función $u(t, x)$, es una expresión de la forma:

$$
\partial_{t} u(t, x)-H\left(t, x, u(t, x), D u(t, x), D^{2} u(t, x)\right)=0
$$


con $x \in \Omega\left(\right.$ domino abierto en $\left.\mathbb{R}^{n}\right), t \in[0, T)$ y condición terminal $u(T, x)=g(x)$. Se tiene que $D u$ denota el gradiente ${ }^{6}$ de la función $u, D^{2} u$ el Hessiano ${ }^{7}$ y $H(\cdot)$ el Hamiltoniano ${ }^{8}$.

Dos casos particulares de (18) son:

- EDP semilineal:

$$
\partial_{t} u(t, x)-\sum_{i=1, j=1}^{n} a_{i j}(t, x) \partial_{i j} u(t, x)=f(t, x, u, D u)
$$

- EDP cuasilineal:

$$
\partial_{t} u(t, x)-\sum_{i=1, j=1}^{n} a_{i j}(t, x, D u) \partial_{i j} u(t, x)=f(t, x, u, D u)
$$

\subsection{Control óptimo estocástico y ecuación de Hamilton-Jacobi- Bellman}

Muchos problemas financieros, como el de valoración de derivados, pueden plantearse como un problema de control óptimo estocástico. El tratamiento estándar para estos consiste en plantear y resolver la ecuación diferencial parcial no lineal de Hamilton-Jacobi-Bellman (HJB) asociada al problema.

Asumiendo que los procesos $X_{t}^{i}$ satisfacen:

$$
d X_{t}^{\alpha, i}=b_{i}\left(t, X_{t}^{\alpha}, \alpha_{t}\right) d t+\sum_{j=1}^{d} \sigma_{i, j}\left(t, X_{t}^{\alpha}, \alpha_{t}\right) d W_{t}^{j} \quad ; \quad i \in\{1, \ldots, n\}
$$

donde los coeficientes de tendencia $\left(b_{i}\right)$ y difusión $\left(\sigma_{i, j}\right)$ dependen de $t, X_{t}$ y de un control $\alpha_{t}$, un problema de control óptimo estocástico estándar asociado a estos procesos busca optimizar (maximizar o minimizar) la función

$6 \quad$ Vector de primeras derivadas.

7 Matriz de segundas derivadas.

8 Función que describe el estado del sistema. 


$$
J(t, x)=E^{Q}\left[\int_{t}^{T} f\left(s, X_{s}^{\alpha}, \alpha_{s}\right) d s+g\left(X_{T}^{\alpha}\right) \mid X_{t}^{\alpha}=x\right]
$$

respecto al control $\alpha$, es decir, se busca determinar la función:

$$
u(t, x):=\sup _{\alpha} J(t, x)
$$

La función $u(t, x)$ en (23) satisface la EDP de HJB

$$
-\partial_{t} u(t, x)-\sup _{\alpha}\left\{A^{\alpha} u(t, x)+f(t, x, \alpha)\right\}=0
$$

Con $u(T, x)=g(x)$

\section{Problemas no lineales de valoración en finanzas}

A continuación se describen algunos de los modelos de valoración en los cuales se eliminan o flexibilizan varios de los supuestos iniciales del modelo BlackScholes, lo que lleva a considerar EDP no lineales.

Volatilidad incierta. En este caso se asume que el precio del activo subyacente $\left(S_{t}\right)$ es una martingala local bajo la medida $Q$, descrita mediante la ecuación diferencial estocástica (por simplicidad se asume que el coeficiente de tendencia en la expresión es igual a cero, sin pérdida de generalidad):

$$
d S_{t}=\sigma_{t} S_{t} d W_{t}
$$

En este caso, la volatilidad del proceso de precio $\left(\sigma_{t}\right)$ es un proceso $F_{t}$ medible (siguiendo el trabajo de Avellaneda et al., 1995) que se asume como evaluado en el intervalo compacto $[\underline{\sigma}, \bar{\sigma}]$, cuyos valores extremos pueden asociarse a momentos de baja y alta volatilidad. En este contexto, el problema de valorar en $t \leq T$ una opción pactada sobre este subyacente, con vencimiento en $T$ y valor intrínseco $F_{T}$, es la solución del problema de control óptimo estocástico: 


$$
u_{t}=\sup _{[t, T]} E^{Q}\left[F_{T} \mid F_{t}\right]
$$

donde el supremo es considerado sobre todos los procesos $\left(\sigma_{s}\right)_{t \leq s \leq T} \operatorname{con} s \in[t, T]$ en el dominio $[\underline{\sigma}, \bar{\sigma}]$. Esta expresión de valoración corresponde al precio de superreplicación del comprador bajo el supuesto de volatilidad incierta.

Asumiendo que el valor intrínseco de la opción es de la forma $F_{T}=g\left(S_{T}\right)$, la EDP de HJB es:

$$
\partial_{t} u(t, x)+\frac{1}{2} \sup _{\sigma \in[\underline{\sigma}, \bar{\sigma}]} \sigma^{2} x^{2} \partial_{x}^{2} u(t, x)=0
$$

Tomando el supremo sobre todo $\sigma$, se tiene una EDP completamente no lineal, denominada EDP de Black-Scholes-Barenblatt, de la forma:

$$
\partial_{t} u(t, x)+\frac{1}{2} x^{2} \sum\left(\partial_{x}^{2} u(t, x)\right)^{2} \partial_{x}^{2} u(t, x)=0 \quad, \quad u(T, x)=g(x)
$$

donde: $\sum(\varphi)=\underline{\sigma} 1_{\varphi<0}+\bar{\sigma} 1_{\varphi \geq 0}$

Costos de transacción. Asumiendo costos de transacción asociados a los rebalanceos necesarios para mantener la cobertura en el derivado, la EDP de valoración es de la forma no lineal:

$$
\partial_{t} u(t, x)+\frac{1}{2} x^{2} \sum\left(\partial_{x}^{2} u(t, x)\right)^{2} \partial_{x}^{2} u(t, x)=0 \quad, \quad u(T, x)=g(x)
$$

donde: $\Sigma(A)^{2}=\sigma^{2}(1-\alpha) \mathbf{1}_{A<0}+\sigma^{2}(1+\alpha) \mathbf{1}_{A \geq 0}$

Mercados ilíquidos. Considerando un mercado ilíquido, el cual es caracterizado mediante el parámetro de elasticidad $\xi$ que está dado por el cociente entre el cambio relativo en el precio de los activos y el cambio en la demanda neta $\left(M_{t}\right)$, es decir:

$$
\frac{d S_{t} / S_{t}}{d M_{t}}=\xi \quad \Rightarrow \quad \frac{d S_{t}}{S_{t}}=\xi d M_{t}
$$


en donde se asume que $\xi$ es una constante no negativa. La relación (30) permite analizar la influencia de las estrategias de negociación dinámica sobre los precios de los derivados financieros, considerando que:

- $\quad M\left(t, W_{t}, S_{t}\right)$ denota la demanda de todos los agentes en el mercado.

- $\frac{d S_{t}}{S_{t}}=\mu\left(t, S_{t}\right) d t+\sigma\left(t, S_{t}\right) d W_{t}$

Aplicando el lema de Itô para caracterizar la dinámica de la demanda se tiene:

$$
\begin{aligned}
d M_{t}=\left(\partial_{t} M+\frac{1}{2} \sigma^{2} S_{t}^{2} \partial_{s}^{2} M+\frac{1}{2} \partial_{w}^{2} M\right. & \left.+\sigma S_{t} \partial_{s w} M+\mu S_{t} M\right) d t \\
& +\left(\sigma S_{t} \partial_{s} M+\partial_{w} M\right) d W_{t}
\end{aligned}
$$

Además, de la expresión (30) se tiene que:

$$
d M_{t}=\frac{1}{\xi} \frac{d S_{t}}{S_{t}}=\frac{1}{\xi}\left(\mu\left(t, S_{t}\right) d t+\sigma\left(t, S_{t}\right) d W_{t}\right)
$$

Igualando las expresiones (31) y (32) se tiene que:

- $\mu(t, x)=\xi \frac{\partial_{t} M+\frac{1}{2} \sigma^{2} x^{2} \partial_{x}^{2} M+\sigma x \partial_{x w} M+\frac{1}{2} \partial_{w}^{2} M}{1-\xi x \partial_{x} M}$

- $\sigma(t, x)=\frac{\xi \partial_{w} M}{1-\xi x \partial_{x} M}$

Para caracterizar el mercado a partir de las expresiones anteriores se consideran dos tipos de agentes, pequeños ( $M$ pequeño) y grandes ( $M$ grande), donde:

$$
M_{\text {pequeño }}=\frac{1}{\xi}\left(\mu_{0} t+\sigma_{0} W_{t}\right) \quad, \quad M_{\text {grande }}=\Delta\left(t, S_{t}\right)
$$

y $M_{\text {Total }}=M_{\text {pequeño }}+M_{\text {grande }}$. De acuerdo con esto se tiene que: 


$$
\begin{gathered}
\mu(t, x)=\frac{\mu_{0} \xi\left[\partial_{t} \Delta+\frac{1}{2} \sigma^{2} x^{2} \partial_{x}^{2} \Delta\right]}{1-\xi x \partial_{x} \Delta} \\
\sigma(t, x)=\frac{\sigma_{0}}{1-\xi x \partial_{x} \Delta}
\end{gathered}
$$

Las expresiones (33) y (34) describen el efecto feedback de las negociaciones dinámicas sobre el retorno y la volatilidad del precio de los activos. En este caso, la función $u(t, x)$ es la solución de EDP de Black-Scholes no lineal

$$
\partial_{t} u(t, x)+\frac{1}{2} x^{2} \sum\left(x, \partial_{x}^{2} u(t, x)\right)^{2} \partial_{x}^{2} u(t, x)=0 \quad, \quad u(T, x)=g(x)
$$

donde: $\sum(x, \Gamma)^{2}=\frac{\sigma_{0}^{2}}{(1+\xi x \Gamma)^{2}}$.

Tasas diferenciales para inversión o préstamo. En este caso se asume que los agentes presentes en el mercado pueden invertir a una tasa $\underline{r}$ y tomar prestado a una tasa $\bar{r}$, con $\underline{r} \leq \bar{r}$. Para determinar el efecto de estas tasas en la valoración se debe considerar la EDP que se tiene para el caso en el cual las tasas de inversión o préstamo son iguales, constantes y conocidas a un valor $r$.

$$
\partial_{t} u(t, x)+\frac{1}{2} \sigma(t, x)^{2} x^{2} \partial_{x}^{2} u(t, x)+r x \partial_{x} u(t, x)-r u(t, x)=0
$$

con $u(T, x)=g(x)$. En esta ecuación, la tasa de interés $r$ explica que sobre el intervalo $[t, t+d t]$ el efectivo se invierte o toma prestado dependiendo del signo de $\partial_{x} u(t, x)$ y del signo (posición) de $u(t, x)$. Por ejemplo, el vendedor de una opción call europea debe prestar dinero para comprar las acciones necesarias a fin de realizar una cobertura delta, lo que hace la opción más costosa y, por eso, el término positivo $r x \partial_{x} u(t, x)$. Adicionalmente, este agente también puede invertir la prima positiva de la opción, lo que hace la opción más barata, de ahí el término - $r u(t, x)$. El efecto neto es que el agente tendría que prestar dinero si $x \partial_{x} u(t, x)-u(t, x) \geq 0$ o invertir si $u(t, x)-x \partial_{x} u(t, x) \geq 0$.

De acuerdo con esto, si se asumen tasas diferenciales para inversión y préstamo, la EDP (36) es reemplazada por: 


$$
\partial_{t} u(t, x)+\frac{1}{2} \sigma(t, x)^{2} x^{2} \partial_{x}^{2} u(t, x)+R\left(x \partial_{x} u(t, x)-u(t, x)\right)=0
$$

donde:

$$
R(y)= \begin{cases}\overline{r y} & \text { si } y \geq 0 \\ r y & \text { si } y<0\end{cases}
$$

Se tiene que la ecuación (37) es semilineal y la expresión:

$$
\sup _{r \in\{\underline{r}, \bar{r}\}}\left(\partial_{t} u(t, x)+\frac{1}{2} \sigma(t, x)^{2} x^{2} \partial_{x}^{2} u(t, x)+r x \partial_{x} u(t, x)-r u(t, x)\right)=0
$$

expresa el problema de valoración bajo tasas diferenciales como un problema de control óptimo estocástico con EDP de HJB dada por (38).

Ajuste por riesgo de crédito. Considerando la posibilidad de que la contraparte del derivado incumpla con sus obligaciones, se incorpora un ajuste en la valoración que incluya el costo de cubrir este escenario. Por simplicidad se asumirá que el riesgo de contraparte es unilateral, es decir que solo una de las partes $(C)$ puede entrar en incumplimiento, mientras que la otra parte $(B)$ no puede hacerlo. Se asumirá que $B$ puede negociar de forma dinámica en $d$ activos subyacentes $S^{1}, S^{2}, \ldots, S^{d}$ y que, adicionalmente, para cubrir su riesgo en $C, B$ puede negociar un bono o título asociado al riesgo de default de $C$ (cds), denotado por $P_{t}^{C}$.

Se considera que los precios de los subyacentes no se alteran por el incumplimiento de la contraparte, que es descrito mediante un proceso Poisson de intensidad constante. Se denota por $u$ al valor de la posición larga de $B$ en el derivado pactado con $C$, asumiendo que hasta el momento de la valoración $C$ no ha incumplido. Por simplicidad se asumen cero dividendos y una tasa de interés constante $r$. Bajo el modelo de volatilidad local, el mercado es completo, y bajo la única medida de riesgo neutral $Q$ los procesos de precio $S_{t}=\left(S_{t}^{1}, S_{t}^{2}, \ldots, S_{t}^{d}\right)$ y $P_{t}^{C}$ satisfacen:

$$
\frac{d S_{t}^{i}}{S_{t}^{i}}=r d t+\sum_{j=1}^{d} \sigma_{i, j}\left(t, S_{t}^{i}\right) d W_{t}^{j}
$$




$$
\frac{d P_{t}^{C}}{P_{t}^{C}}=\left(r+\lambda_{C}\right) d t-d J_{t}^{C}
$$

siendo $W_{t}$ un movimiento Browniano $d$-dimensional y $J_{t}^{C}$ un proceso Poisson con intensidad $\lambda_{C}$. Como el mercado es completo, la condición de no arbitraje establece que $e^{-r t} u\left(t, S_{t}\right)$ es una $Q$-martingala caracterizada por:

$$
\partial_{t} u+A u+\lambda_{C}(\bar{u}-u)-r u=0 \quad, \quad u(T, x)=g(x)
$$

donde: $A$ es el operador de Itô de $S_{t}$ y $\bar{u}$ es el valor del derivado justo después del incumplimiento de la contraparte. En el evento de incumplimiento $\bar{u}$ está dado por $\bar{u}=R M^{+}-M^{-}$, donde $M$ es el valor de mercado del derivado y $R$ es la tasa de recuperación en caso de incumplimiento. Se tiene entonces que, si $M$ es positivo $B$ debería recibir de $C$ el monto $M$, y solo la fracción $R M$ en caso de incumplimiento de $C$. Si $M$ es negativo $B$ debería entregar a $C$ el monto $M$, y este será pagado en caso de incumplimiento de $C$.

Si el riesgo de incumplimiento es bilateral la EDP (41) es reemplazada por:

$$
\partial_{t} u+A u+\lambda_{C}\left(\bar{u}_{C}-u\right)+\lambda_{B}\left(\bar{u}_{B}-u\right)-r u=0
$$

con $\bar{u}_{B}=M^{+}-R M^{-}$y $\lambda_{B}$ la intensidad del proceso Poisson de incumplimiento de $B$.

No hay consenso en el mercado acerca de la inclusión o no de la provisión por riesgo de contraparte para la liquidación del valor del derivado en caso de incumplimiento. Se tiene entonces que:

1. Si se considera la provisión por el riesgo de contraparte, $M=u$ entonces:

$$
\partial_{t} u+A u-(1-R) \lambda_{C} u^{+}-r u=0 \quad ; \quad u(T, x)=g(x)
$$

2. No se considera la provisión por el riesgo de contraparte,

$$
\left.M=e^{-(r+(1-R) \lambda} C\right)(T-\mathrm{t}) E_{t, x}\left[g\left(X_{T}\right)\right]
$$


y en este caso:

$$
\partial_{t} u+A u+\left(R M^{+}-M^{-}-u\right)-r u=0 \quad ; \quad u(T, x)=g(x)
$$

Al descontar y reemplazar $u$ por $-u$, para mantener más clara la presentación, las EDP (43) y (44) se pueden llevar a la forma estándar:

$$
\partial_{t} u+A u+\beta\left(u^{+}-u\right)=0
$$

con $\beta=\lambda_{C}(1-R)$ y condición terminal $u(T, x)=g(x)$, que resulta ser una EDP no lineal.

\section{Conclusiones y extensiones}

El objetivo de este documento era presentar una introducción al problema de la valoración no lineal de derivados financieros, donde la no linealidad está determinada por la eliminación de algunos de los supuestos del modelo básico Black-Scholes de valoración.

En las secciones anteriores se realizó la descripción de algunas de las extensiones más comunes del modelo base desde la perspectiva de las ecuaciones diferenciales parciales, y en todos los casos estudiados se muestra que el problema de valoración lleva a una ecuación diferencial parcial no lineal, lo que abre la puerta a la pregunta de cómo resolver este tipo de ecuaciones de forma eficiente.

En futuras publicaciones se estudiarán dos aproximaciones al problema. La primera es una extensión del teorema de Feynman-Kac al caso no lineal, que utiliza ecuaciones diferenciales estocásticas hacia atrás (BSDE por sus siglas en inglés), siguiendo trabajos como los de El Karoui, Peng y Quenez (1997) o Pardoux y Peng (1990). La segunda es un método numérico presentado por Guyon y Henry-Labordére (2013) que utiliza difusiones ramificadas.

Se espera que este primer documento sirva de motivación al lector para continuar con el tema propuesto, ya que este es un interesante campo de confluencia de la ciencia financiera, las matemáticas, la probabilidad, los métodos numéricos y la computación para resolver problemas concretos de los mercados. 


\section{Referencias}

Avellaneda, M., Levy, A., y Parás, A. (1995). Pricing and hedging derivative securities in markets with uncertain volatilities. Applied Mathematical Finance, 2(2), 73-88.

Benhamou, E., Rivoira, A., y Gruz, A. (2008). Stochastic interest rates for local volatility hybrids models. Wilmott magazine.

Black, F., y Scholes, M. (1973). The pricing of options and corporate liabilities. Journal of Political Economy, 81(3), 637-654.

Carmona, R., y Nadtochiy, S. (2009). Local volatility dynamic models. Finance and Stochastics, 13(1), 1-48.

Cetin, U., Jarrow, R. A., y Protter, P. (2004). Liquidity risk and arbitrage pricing theory. Finance and Stochastics, 8(3), 311-341.

Cetin, U., Soner, H. M., y Touzi, N. (2010). Option hedging for small investors under liquidity costs. Finance and Stochastics, 14(3), 317-341.

Cox, J. C., Ross, S. A., y Rubinstein, M. (1979). Option pricing: A simplified approach. Journal of Financial Economics, 7(3), 229-263.

El Karoui, N., Peng, S., y Quenez, M. C. (1997). Backward stochastic differential equations in finance. Mathematical Finance, 7(1), 1-71.

Forsyth, P. A., y Vetzal, K. R. (2001). Implicit solution of uncertain volatility/transaction cost option pricing models with discretely observed barriers. Applied Numerical Mathematics, 36(4), 427-445.

Guyon, J., y Henry-Labordère, P. (2013). Nonlinear option pricing. CRC Press.

Kampen, J., y Avellaneda, M. (2003). On parabolic equations with gauge function term and applications to the multidimensional leland equation. Applied Mathematical Finance, 10(3), 215-228.

Leland, H. E. (1985). Option pricing and replication with transactions costs. The Journal of Finance, 40(5), 1283-1301. 
Lyons, T. J. (1995). Uncertain volatility and the risk-free synthesis of derivatives. Applied Mathematical Finance, 2(2), 117-133.

Martínez, F. V. (2008). Riesgos financieros y economicos/Financial and economical risks: Productos derivados y decisiones economicas bajo incertidumbre. Cengage Learning Editores.

Moreno Trujillo, J. (2015). Modelos estocásticos en finanzas. Bogotá: Universidad Externado de Colombia.

Pardoux, E., y Peng, S. (1990). Adapted solution of a backward stochastic differential equation. Systems \& Control Letters, 14(1), 55-61. 